\title{
Effects of a direct-fed microbial and feedlot pen environmental change on phenotypic resistance among enterococci isolated from beef cattle feces
}

\author{
Sarah A. Murray ${ }^{1 \wedge}$, Ashlyn C. Holbert ${ }^{2}$, Keri N. Norman $^{2}$, Sara D. Lawhon ${ }^{1}$, Jason E. Sawyer ${ }^{3 \sharp}$, Harvey M. Scott $^{1 *}$ \\ 1 Department of Veterinary Pathobiology, Texas A\&M University, College Station, TX 77843, USA; $\underline{\text { smur- }}$ \\ ray@cvm.tamu.edu (S.A.M.); slawhon@cvm.tamu.edu (S.D.L.) \\ 2 Department of Veterinary Integrative Biosciences, Texas A\&M University, College Station, TX 77843, USA; \\ aholbert@cvm.tamu.edu (A.C.H); knorman@cvm.tamu.edu (K.N.N) \\ 3 Department of Animal Science, Texas A\&M University, College Station, TX 77843, USA; j-sawyer@tamu.edu \\ Current Address: United States Department of Agriculture-Agricultural Research Service, Meat Animal \\ Research Center, Clay Center, NE 68933 USA; sarah.murray@usda.gov \\ \# Current Address: King Ranch Institute for Ranch Management, Texas A\&M University, Kingsville, TX \\ 78363, USA; jason.sawyer@tamuk.edu \\ * Correspondence: $\underline{\text { hmscott@cvm.tamu.edu; }}$ Tel.: +1-(979)-847-6197
}

\begin{abstract}
In two sequential replicates ( $\mathrm{n}=90$ and $\mathrm{n}=96$ feedlot finisher cattle, respectively) we measured the impact of an Enterococcus faecium-based probiotic (DFM) and an altered feedlot pen environment on antimicrobial resistance among fecal enterococci in cattle fed (or, not fed) the macrolide tylosin. Diluted fecal samples were spiral-plated on plain and antibiotic-supplemented m-Enterococcus agar. In the first replicate, tylosin significantly $(\mathrm{p}<0.05)$ increased the relative quantity of erythromycin-resistant enterococci. This effect was diminished in cattle fed the DFM in conjunction with tylosin. A similar observed effect was not statistically significant $(P>0.05)$ in the second replicate. Isolates were speciated and resistance phenotypes were obtained for E. faecium and E. hirae. E. faecium isolates were whole-genome sequenced, which yielded sequence types (ST), resistance genes and phylogeny. Samples of the DFM were sequenced and found to contain E. faecium ST296, which was not present on Day 0 of either replicate. This DFM sequence type was found in fecal samples after Day 0, the majority of which were isolated from cattle in one of the DFM-fed pens. Increased prevalence of ST296 occurred with a concomitant decrease in ST240; of importance, the latter typically harbored both ermB and tet( $(\mathrm{M})$ genes.
\end{abstract}

Keywords: probiotic; Enterococcus faecium; antimicrobial resistance; environmental change

\section{Introduction}

In both human and veterinary medicine, antimicrobial resistance is rapidly becoming a worldwide crisis. In 2019, the U.S. Centers for Disease Control and Prevention (CDC) released an updated version of their 2013 document called: "Antibiotic Resistance Threats in the United States", which compiled a comprehensive list of the top AMR threats [1,2]. This updated CDC report stated that at least 2.8 million resistant bacterial infections occur annually in the United States, resulting in over 35,000 deaths. In 2015, the World Health Organization (WHO) released its Global Action Plan to combat antibiotic resistance, designed to be implemented in collaboration with the Food and Agriculture Organization of the United Nations (FAO) and the World Organization for Animal Health (OIE) under a "Tripartite" agreement [3]. The second objective in the WHO plan includes: "...research to identify alternatives to nontherapeutic uses of antimicrobial agents in agriculture and aquaculture, including their use for growth promotion and crop protection". The WHO Global Action Plan complements both the 2012 and 2013 
U.S. Food and Drug Administration Center for Veterinary Medicine (FDA-CVM) Guidances for Industry \#209 and \#213, respectively, which outlined judicious use of antibiotics (including restricting growth-promotion uses) and recommendations for new product uses [4,5]. Additionally, in 2019 the FDA-CVM released a draft Guidance for Industry \#263, which outlined implementation of veterinary oversight for all medically-important antimicrobial use in animals, effectively ending the last loophole for non-veterinary oversight sales as over-the-counter drugs for parenteral and systemic use [6].

While growth promotion use is no longer allowed in the United States since 2016, antibiotics are still widely used for prevention, control, and treatment of disease. Antibiotics, including tylosin and chlortetracycline, are used in feed for the prevention and control of liver abscesses in cattle. High grain diets are widely accepted as the main cause of ruminal acidosis, rumenitis, and subsequent liver abscess formation $[7,8]$. Though it remains unknown as to any one specific causative agent and the exact pathogenesis for liver abscesses, Fusobacterium necrophorum (previously known as Sphaerophorus necrophorus, Fusiformis necrophorus, and Bacillus necrophorus) is commonly isolated from abscessed livers, as is Trueperella pyogenes (previously known as Actinomyces pyogenes). It has been suggested that these two organisms initiate a synergistic reaction in causing the formation of liver abscesses [9]. Recently, certain Salmonella enterica serotypes such as Lubbock have been isolated during anaerobic culture from a liver abscess $[7,10]$.

Liver abscesses in cattle result in diminished carcass value and are responsible for large economic losses in the fed beef cattle industry [11,12]. Cattle with severe liver abscesses may require more carcass trimming because of adhesion of the abscesses to the diaphragm and surrounding peritoneal cavity and organs. In some instances, condemnation of the entire viscera may be necessary. Abscesses are the leading cause of liver condemnation, ranking second in the top 10 concerns of packers [8]. Additionally, the accidental rupture of an abscess and contamination of a carcass with pus can cause interruption in the flow of carcasses along the chain on the slaughter floor, thus costing time and labor.

That said, the greatest economic impact of liver abscesses results from reduced animal performance and decreased carcass yield. Cattle with abscessed livers have reduced feed intake, reduced weight gain, decreased feed efficiency, and decreased carcass dressing percentage [13]. Feed intake and feed conversion are impacted by severe liver abscesses, reducing intake by $5 \%$ and gain-to-feed by $14 \%$ [13]. A study by Brown et al. [14] also reported that adhesions increased the loss in HCW (hot carcass weight) by $3 \mathrm{~kg}$ in one comparison, and by $8.7 \mathrm{~kg}$ in a second comparison. They also reported a reduction in marbling score, in addition to reductions in yield grade, fat depth, and percent of kidney-pelvic-heart (KPH) fat in cattle with severe liver abscesses, versus cattle with normal livers [11]. Additionally, a meta-analysis on liver abscess risks of cattle receiving tylosin versus cattle not receiving tylosin in conventional feeding systems showed that the feeding of tylosin reduced the risk of liver abscesses from $30 \%$ to $8 \%$ [15].

Meanwhile, tylosin (a macrolide antibiotic containing a 16-membered lactone ring) has been shown to select for macrolide resistance when used as a growth promoter in swine [16]. In cattle, tylosin has also been associated with increased resistance to macrolides among fecal enterococci $[17,18]$. A systematic review of tylosin use in cattle estimated that when fed at approved dosages for typical durations, tylosin tended to increase the proportion of macrolide-resistant enterococci in cattle, thus suggesting a potential zoonotic risk to human beef consumers [19]. Additionally, in recent surveillance of all enterococci across the One Health continuum, Enterococcus hirae was the most common species isolated from cattle, followed by Enterococcus villorum and then Enterococcus faecium [20]. Furthermore, resistance to tetracycline and macrolides appeared abundant among the 
majority of enterococcal species which the author posited as likely being due to the common use of antibiotics in both human and veterinary medicine [20].

This is of importance because Enterococcus faecium, though a common commensal bacterium, also is recognized as a leading opportunistic cause of nosocomial infections in intensive human health care settings [21]. in fact, E. faecium has been noted as being the second most prevalent nosocomial pathogen [22,23]. In the 2019 updated version of the Antimicrobial Resistance Threats Report by the U.S. Centers for Disease Control and Prevention, E. faecium was identified as the most common cause of central line associated bloodstream infections [2]. While E. faecium is less likely to possess virulence factors than E. faecalis, it is more likely to carry a multi-drug resistance geno- and phenotype [24]. Remarkably, when the phylogeny of multidrug-resistant E. faecium was traced, it was found that while the emergence of the hospital-adapted lineage occurred in association with the early human use of antibiotics, the bacterial populations at that time consisted of a majority of animal-derived strains and was not associated with human commensals [25]. The same authors traced an earlier bifurcation of E. faecium, the divergence of human and animal strains (3,000+ years ago) to a time corresponding with the emergence of domestic agriculture, including the keeping of livestock and specialized diets.

Therefore, consideration of the possibility and impacts of shared mobile genetic elements among host-adapted strain of enterococci must be made. A problem arises when examining resistance to erythromycin in association with feeding tylosin, because it has been associated with erm family of genes which confer resistance to a wide variety of macrolides, lincosamides, and streptogramin B. $[17,18]$. Additionally, there is the possibility of co-selection of resistance to tetracycline when feeding tylosin, and vice versa [26]. Furthermore, macrolides such as erythromycin and azithromycin are a key antibiotics in human health care, deemed to be in a category of the highest priority and of critical importance by the World Health Organization [27].

In response to these concerns, several studies have been performed exploring ways to limit tylosin use, or else to find non-antibiotic alternatives for liver abscess prevention. In 2015, Beukers et al. [28] suggested that antibiotic withdrawal prior to slaughter contributed to a reduction in the proportion of macrolide-resistant enterococci. Additionally, in 2018, Muller et al. [29] showed no difference in resistance among fecal enterococci from cattle fed intermittent tylosin supplementation versus continuous treatment; thereby, suggesting that environmental factors may be most important in carrying over resistance from one lot of cattle to the next, even more so than contemporaneous selection occurring during the actual feeding period. In another direction, Saccharomyces cerevisiae fermentation products (SCFP) have been suggested for use to prevent liver abscesses, though there have been no statistically significant differences reported among treatment groups with respect to abscess prevalence or severity scores [30]. Enterococcus faecium is a unique bacterium in that it can be deployed as a probiotic, especially due to its bile tolerance and its bacteriocins which are antagonistic towards pathogenic organisms such as Listeria monocytogenes [31]. Therefore, by studying both the independent and interactive combined effects of an Enterococcus faecium-based direct-fed microbial (probiotic), tylosin and pen environmental change on resistance in enterococci, we report their effects on $\log _{10}$ overall and resistant Enterococcus CFU, phenotypic multidrug resistance of select isolates, and trends in resistance to several other antibiotics.

\section{Materials and Methods}

\subsection{Experimental Design}

A randomized and controlled field trial consisting of two serial replicates in a $2 \times 2 \times 2$ factorial design was conducted at the Texas A\&M Agri-Life Research experimental feedlot in McGregor, Texas (AACUC AUP \#2015-026A; IBC \#2017-049 and \#2017-021). This 
facility was unique in that it previously had 8 cattle pens in use for many years; in addition, 8 new pens were purpose-built for this study in which no antibiotics had ever been used or fed, or housed animals that had previously been treated with any antibiotic. Antibiotic-free and grass-fed beef cattle were allowed access to the new pens to prepare a manure pack during the 4 weeks prior to the trial; importantly, this was designed to ensure that a homogenous baseline of fecal bacteria was present in the newly constructed pens prior to the beginning of the feeding trial. The DFM used (Tri-Lution, Agri-King, Fulton, IL) contained $1.3 \times 10^{7} \mathrm{CFU} / \mathrm{g}$ of Enterococcus faecium and Saccharomyces cerevisiae. The DFM was top-dressed into the feed for the proper treatment group. At the onset of the trial, steers were placed in the old pens and randomly assigned to a treatment group; treatments being: 1) tylosin (Tylan, Elanco, Greenfield, IN) included at 7.3g/tonne), 2) DFM (824.5g/tonne), 3) both tylosin and DFM, and 4) neither tylosin nor DFM (control). These four treatment groups were repeated in serial duplicates, so each treatment group had a replicate (Figure 1A). Four weeks prior to slaughter (hereafter, referred to as the withdrawal timepoint), tylosin was removed from the respective trial ration while keeping the DFM feeding regimen for the appropriate treatment groups; meanwhile, half the steers in each of the four treatment groups were randomly assigned to a newly constructed adjacent pen purpose-built for this study (Figure 1B). 

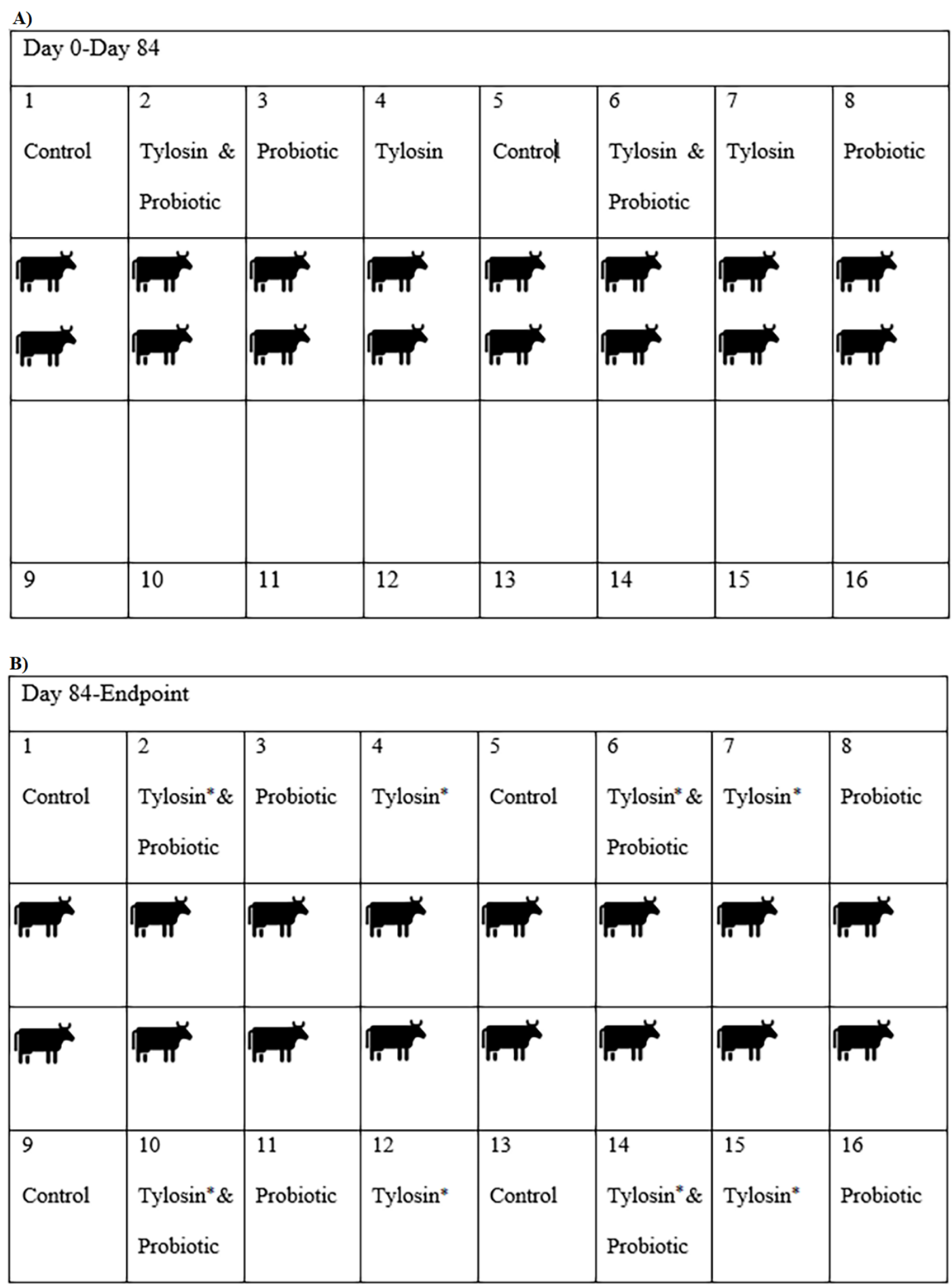

Figure 1 A) Pen layout for first 12 weeks. Each cartoon figure represents six cattle. B) Pen layout for last 4 weeks. Pens 1-8 were the 'old' pens, Pens 9-16 were the purpose-built 'new' pens. *Cattle were not fed tylosin from this point forward

The first replicate consisted of 90 steers, while the second replicate consisted of 96 steers, all sourced from the same ranch birth cohort. Once every 28 days, in the morning, fecal samples were collected per rectum using new individual rectal palpation sleeves for 3 months (i.e., from Day 0 to Day 84) by the McGregor, Texas feedlot crew. Following Day 84 , starting at the tylosin withdrawal time point, samples were taken weekly until slaughter. Samples were transported directly to the laboratory on ice immediately following the 
completion of collection. Samples were stored in the refrigerator until the next day, at which time they were processed. Sample processing consisted of aliquoting fecal samples into two $5 \mathrm{ml}$ tubes; specifically, into one tube without glycerol and one tube with sterile $50 \%$ glycerol at a 1:1 ratio of glycerol to feces. Tubes were preserved at $-80^{\circ} \mathrm{C}$ until further use. This sample collection schedule, processing scheme, and storage was repeated for each of the two trial replicates.

\subsection{Bacterial enumeration, isolation, and speciation}

Samples from Day 0 as the pretrial baseline measure, Day 84 as the antibiotic withdrawal time point (i.e., presumed maximum cumulative effect), and slaughter as the final time point, were used for these analyses. Samples preserved with glycerol were thawed on ice and mixed thoroughly with phosphate buffered saline (PBS) (Gibco Life Technologies, Thermo Scientific Microbiology, Oakwood Village, $\mathrm{OH}$ ) using 4.5 milliliters of PBS to 0.5 grams of feces to create a 1:10 dilution. An aliquot of 50 microliters of this dilution was spiral-plated to plain m-Enterococcus agar (Difco, Becton Dickinson Sparks, MD), and to $\mathrm{m}$-Enterococcus agar supplemented with tetracycline and erythromycin at their Clinical and Laboratory Standards Institute (CLSI) human clinical breakpoints of $16 \mathrm{mg} / \mathrm{L}$ and 8 $\mathrm{mg} / \mathrm{L}$, respectively, using an EddyJet 2 Spiral Plater (Neutec Group Inc, Farmingdale, NY). Plates then were incubated at $42^{\circ} \mathrm{C}$ for 48 hours.

Colony counts on each plate were performed using the Flash \& Go® System (Neutec Group Inc, Farmingdale, NY). Two colonies presumptive for Enterococcus faecium (i.e., dark red to maroon with a cream halo) were selected from each of plain and erythromycinsupplemented plate when possible. When presumptive Enterococcus faecium isolates were not available, presumptive Enterococcus hirae was selected instead. Colonies were quadrant streaked for isolation to Tryptic Soy Agar (TSA) with 5\% sheep blood agar (Remel ${ }^{\mathrm{TM}}$, Lenexa, KS) and incubated at $37^{\circ} \mathrm{C}$ for $24-48$ hours. A single colony from each TSA agar with $5 \%$ sheep blood plate was again isolated and streaked fresh to TSA with 5\% sheep blood agar and incubated at $37^{\circ} \mathrm{C}$ for $24-48$ hours and then saved for further analysis.

Each saved isolate was subjected to MALDI-TOF for confirmation of genus and species. Using a new sterilized wooden toothpick per isolate, a single isolate of presumptive Enterococcus faecium or Enterococcus hirae, was spread onto two wells of a reusable 96 well target plate (Bruker Daltonik GmbH., Billerica, MA). Once dry, one microliter of 70\% formic acid was added to the first well of each sample spot pair of each Enterococcus spp. isolate, in addition to one empty spot to serve as a negative control. One microliter of the bacterial test standard (BTS) solution (Bruker Daltonik GmbH., Billerica, MA) was applied to the first and second wells as a positive control. After drying of all wells, one microliter of HCCA matrix solution (Bruker Daltonik GmbH., Billerica, MA) was added to each well, including all the sample wells, BTS wells, formic acid negative control well, and an additional empty well as a secondary negative control. The target plate was then transferred to the MALDI-TOF Microflex LT/SH for reading, using MBT Compass v1.4 software.

\subsection{Phenotypic susceptibility testing}

To obtain minimum inhibitory concentrations, microbroth dilution using the Sensititre® (TREK, Thermo Scientific Microbiology, Oakwood Village, OH) platform was used. Isolates were freshly plated to TSA with $5 \%$ sheep blood agar and incubated at $37^{\circ} \mathrm{C}$ for $18-24$ hours. Afterward, $11 \mathrm{ml}$ of sterilized water was normalized to a 0.5 McFarland standard. Next, $10 \mu \mathrm{l}$ of the culture suspension was transferred to $11 \mathrm{ml}$ of sterile MuellerHinton broth. Subsequently, $50 \mu \mathrm{l}$ of the broth culture was inoculated to each well of the NARMS gram-positive CMV3AGPF plate for Enterococcus spp. using the Sensititre ${ }^{\circledR}$ automated inoculation delivery system (TREK, Thermo Scientific Microbiology, Oakwood Village, $\mathrm{OH})$. The plate consisted of 16 antibiotics from 13 classes, including: chloramphenicol, ciprofloxacin, daptomycin, erythromycin, gentamicin, kanamycin, lincomycin, linezolid, nitrofurantoin, penicillin, quinupristin/dalfopristin, streptomycin, tetracycline, tigecycline, tylosin, and vancomycin (Table 1 ).

Plates were incubated at $37^{\circ} \mathrm{C}$ for 24 hours, with Escherichia coli ATCC 25922, Escherichia coli ATCC 35218, Pseudomonas aeruginosa ATCC 27853, Staphylococcus aureus ATCC 
29213, and Enterococcus faecalis ATCC 29212 serving as quality controls. Plates were read using a Sensititre OptiRead ${ }^{\mathrm{TM}}$ instrument (TREK, Thermo Scientific Microbiology, Oakwood Village, OH). The results were interpreted as susceptible (S), intermediate (I), or resistant (R) in accordance with CLSI guidelines according to the M100 document [32], and NARMS breakpoints when CLSI breakpoints were not available, using SWIN software (TREK, Thermo Scientific Microbiology, Oakwood Village, OH).

Table 1 Antibiotics ordered by class, concentration ranges (mg/L) and interpretive breakpoints (for resistance) for the NARMS (National Antimicrobial Resistance Monitoring System) grampositive plate (CMV3AGP), using CLSI (Clinical and Laboratory Standards Institute) criteria and NARMS interpretive human breakpoints when a CLSI equivalent was unavailable.

\begin{tabular}{cccc}
\hline Antibiotic & Class & Range & Breakpoint \\
\hline Gentamicin & Aminoglycoside & $128-1024$ & $\geq 500$ \\
Kanamycin & Aminoglycoside & $128-1024$ & $\geq 1024$ \\
Streptomycin & Aminoglycoside & $512-2048$ & $>1000$ \\
Vancomycin & Glycopeptide & $0.25-32$ & $\geq 32$ \\
Tigecycline & Glycylcycline & $0.015-0.5$ & $\geq 0.5$ \\
Lincomycin & Lincosamide & $1-8$ & $\geq 8$ \\
Daptomycin & Lipopeptide & $0.25-16$ & $\geq 8$ \\
Erythromycin & Macrolide & $0.25-8$ & $\geq 8$ \\
Tylosin & Macrolide & $0.25-32$ & $\geq 32$ \\
Nitrofurantoin & Nitrofuran & $2-64$ & $\geq 128$ \\
Linezolid & Oxazolidinone & $0.5-8$ & $\geq 8$ \\
Penicillin & Penicillin & $0.25-16$ & $\geq 16$ \\
Chloramphenicol & Phenicol & $2-32$ & $\geq 32$ \\
Ciprofloxacin & Quinolone & $0.12-4$ & $\geq 4$ \\
Quinupristin/dalfopristin & Streptogramin & $0.5-32$ & $\geq 4$ \\
Tetracycline & Tetracycline & $1-32$ & $\geq 16$ \\
\hline
\end{tabular}

\subsection{Statistical Analysis}

CFU bacterial count data were $\log _{10}$ transformed and used in $2 \times 2 \times 2$ full factorial multilevel mixed effect linear regression models, factors being: 1) tylosin, 2) DFM, and 3) sample day with trial replicate and original pen number treated as nested random effects. Due to the low percentage of growth on erythromycin-supplemented agar plates, a Cragg hurdle regression model was used for analysis of the $\log _{10}$ CFU per gram of feces for erythromycin resistant enterococci. For statistical analysis of phenotypic resistance using Sensiti$\operatorname{tre}^{\mathrm{TM}}$, isolates interpreted as intermediate were reclassified as susceptible yielding a binary variable (R/S). Significant increase or decrease in resistance to individual classes of antibiotics was examined using a 2x2x2 full factorial multilevel mixed effect logistic regression model, with factors being: 1) tylosin, 2) DFM, and 3) sample day, and with trial replicate and original pen as nested random effects.

\section{Results}

\subsection{Descriptive Statistics}

Of 558 total fecal samples, 270 samples were collected from Trial Replicate 1 and 288 fecal samples were collected from Trial Replicate 2. From Replicate 1, 98.5\% ( $n=266$ ) of samples were quantifiable on plain m-Enterococcus agar, while $83.7 \%(n=226)$ of samples were quantifiable on tetracycline-supplemented m-Enterococcus, and $31.1 \%(\mathrm{n}=84)$ of samples were quantifiable on erythromycin-supplemented agar. In Replicate 2, 99.3\% ( $\mathrm{n}=286)$ of samples were quantifiable on plain $\mathrm{m}$-Enterococcus agar, while $90.3 \%(\mathrm{n}=260)$ of samples were quantifiable on tetracycline-supplemented m-Enterococcus, and $49.0 \%$ ( $n=141)$ of samples were quantifiable on erythromycin-supplemented agar. The CFU per gram of 
feces was normalized using a $\log _{10}$ transformation. For statistical analyses, samples exhibiting no growth were recorded as zero (0). Samples which were not quantified exhibited no growth (no samples were coded as too numerous to count-TNTC).

\subsection{Mixed Multivariable Regression Models}

For the quantification of total enterococci, a multi-level linear regression was performed on the $\log _{10}$ CFU per gram of feces colony counts, in a $2 \times 2 \times 2$ full factorial design, factors being tylosin, DFM and sample day, with pen and replicate as random effects.

Period effects significantly impacted the $\log _{10} \mathrm{CFU}$ per gram of feces from Day 0 to Day 84 among the DFM, tylosin, and combined DFM/tylosin groups. The DFM group, tylosin group, and combined DFM/tylosin group significantly $(\mathrm{P}<0.05)$ decreased in $\log _{10}$ CFU per gram of feces from Day 0 to Day 84 (Figure 2A), however the control tended to decrease though not significantly different from Day 0. By subtracting the $\log _{10}$ growth on tetracycline-supplemented agar from the corresponding growth on plain agar, the resulting difference is interpretable as follows: the size of the difference is inversely related to levels of antibiotic resistance such that a decrease in the difference between plain and tetracycline-supplemented agar should be interpreted as an increase in resistance. The difference between plain and tetracycline-supplemented agar illustrated a decrease in the proportion of resistance for the DFM group (Figure 2B); however, while this indicates a decrease in resistance, it was not statistically significant $(P>0.05)$. For growth on erythromycin-supplemented agar, a Cragg's hurdle model was used to account for the high number of zero counts (Figure 2C). The DFM group tended to have slightly lower counts on erythromycin-supplemented agar but was not significantly different from any other treatment group. Additionally, none of the treatment-specific temporal changes were statistically significant $(\mathrm{P}>0.05)$.

Comparing the difference between plain and erythromycin-supplemented agar, in the first replicate alone there was a significantly decreased difference $(\mathrm{P}<0.05)$, and therefore increased resistance, in the tylosin group on Day 84 as compared to Day 0 (Figure 2D). Additionally, on Day 84 the tylosin group was significantly different $(\mathrm{P}<0.05)$ from the DFM group. In contrast, the combined tylosin/DFM group was not significantly different from the tylosin group, the DFM group, or the control on Day 84. However, on Day 112,4 weeks after half the cattle were moved to new pens and the withdrawal of tylosin from feed, the tylosin-fed group still showed a significantly decreased difference between plain and erythromycin-supplemented agar when compared to Day 0; however, it was not significantly different from Day 84, and likewise was not significantly different $(\mathrm{P}>0.05)$ compared to any of the other treatment groups on Day 112. When both replicates were combined, treating both pen and replicate as random effects, there remained a tendency towards a decreased difference in CFU on plain and erythromycin plates, and therefore increased levels of resistance, in the tylosin group on Day 84 (Figure 2E). However, this decreased difference was not statistically significant ( $P>0.05)$. Additionally, when both replicates were combined, the tylosin group was not significantly different from the DFM group on Day 84. 

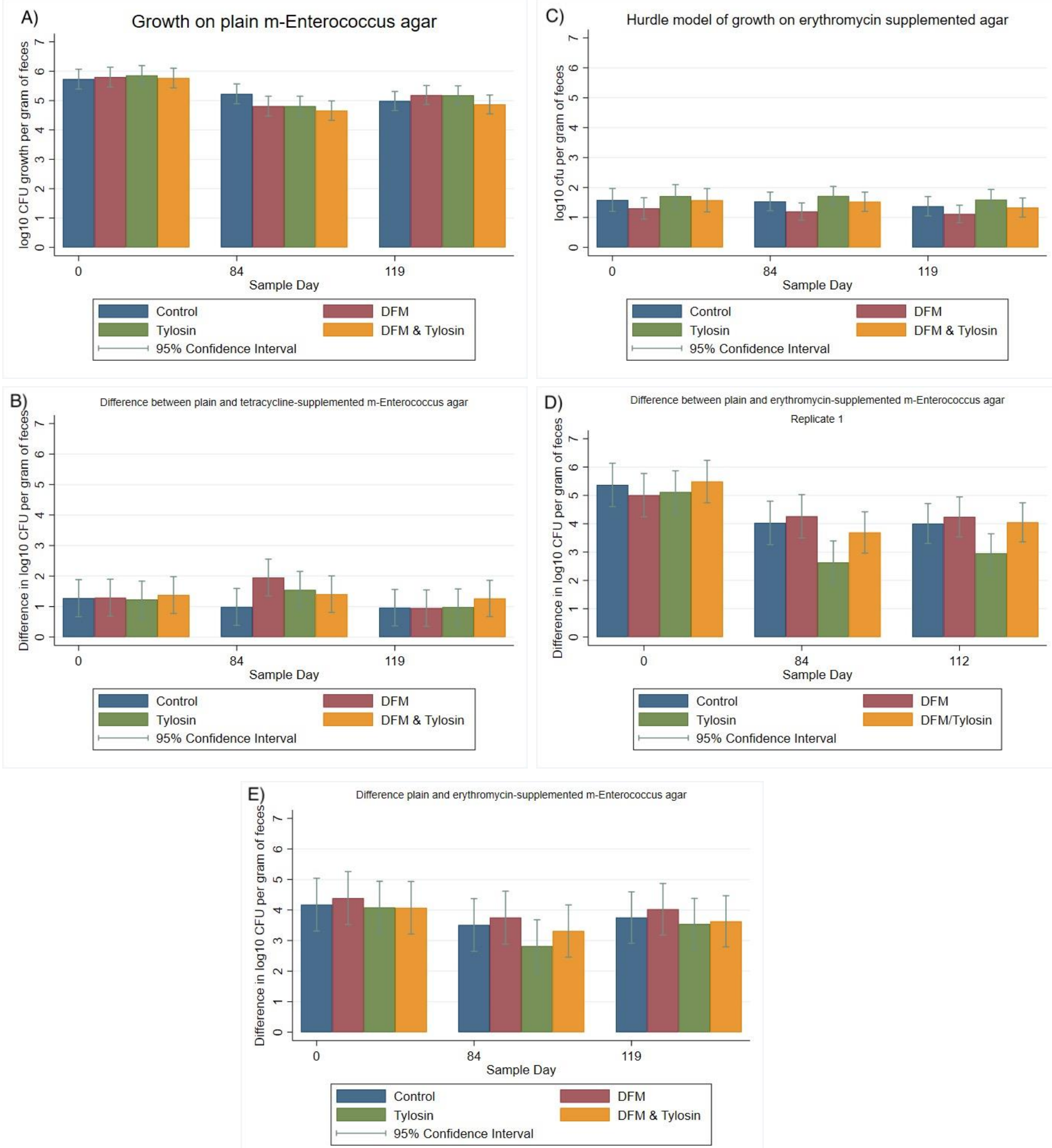

Figure 2 A) Mixed-model marginal mean $\log _{10}$ CFU per gram of feces on plain m-Enterococcus agar, B) Mixed model difference in $\log _{10} \mathrm{CFU}$ between plain and tetracycline supplemented $\mathrm{m}$-Enterococcus agar, C) Cragg hurdle model estimates of $\log _{10}$ CFU per gram of feces on erythromycin supplemented m-Enterococcus agar, D) Mixed model estimated differences in $\log _{10}$ CFU between plain and erythromycin supplemented m-Enterococcus agar from Replicate 1, and E) Mixed model estimated differences in $\log _{10}$ CFU between plain and erythromycin supplemented m-Enterococcus agar from both replicates 


\subsection{Descriptive statistics of phenotypic resistance}

For phenotypic resistance of Enterococcus spp. isolates, all 693 of either E. faecium or E. hirae isolated from plain $\mathrm{m}$-Enterococcus agar, were susceptible to gentamicin, linezolid, tigecycline, and vancomycin (Figure 3). A majority of isolates, $73.3 \%$, were resistant to tetracycline. Additionally, over $50 \%$ of the tetracycline-resistant isolates were right-censored with respect to minimum inhibitory concentration (MIC) and thus continued to grow at the highest concentration of $32 \mathrm{mg} / \mathrm{L}$. Over half of the isolates, a total of $59.6 \%$, were resistant to lincomycin. Only $11.5 \%$ of isolates were resistant to erythromycin. Less than $25 \%$ of the isolates were resistant to daptamycin or nitrofurantoin. Less than $10 \%$ of isolates were resistant to chloramphenicol, ciprofloxacin, kanamycin, penicillin, streptomycin, quinupristin/dalfopristin, or tylosin. Of the $8.7 \%$ of isolates specifically resistant to tylosin, $7.7 \%$ grew at the highest concentration of $32 \mathrm{mg} / \mathrm{L}$, and were therefore right-censored with respect to MIC.

Resistance of Enterococcus spp. isolates to each antibiotic class by sample day and treatment showed a trend towards increasing resistance to macrolides in the tylosin group and the combination tylosin/DFM group (Figure 4). Macrolide resistance decreased in both tylosin-fed groups (following withdrawal) from Day 84 to Day 119. Additionally, there was decreased resistance to tetracycline among isolates in the DFM group and combination tylosin/DFM group. However, the tylosin group increased in resistance from Day 0 to Day 84 , and subsequently decreased after tylosin withdrawal. The increase in macrolide resistance, and the pattern of both increased and decreased resistance to tetracycline was later tested for statistical significance using multi-level mixed logistic regression.

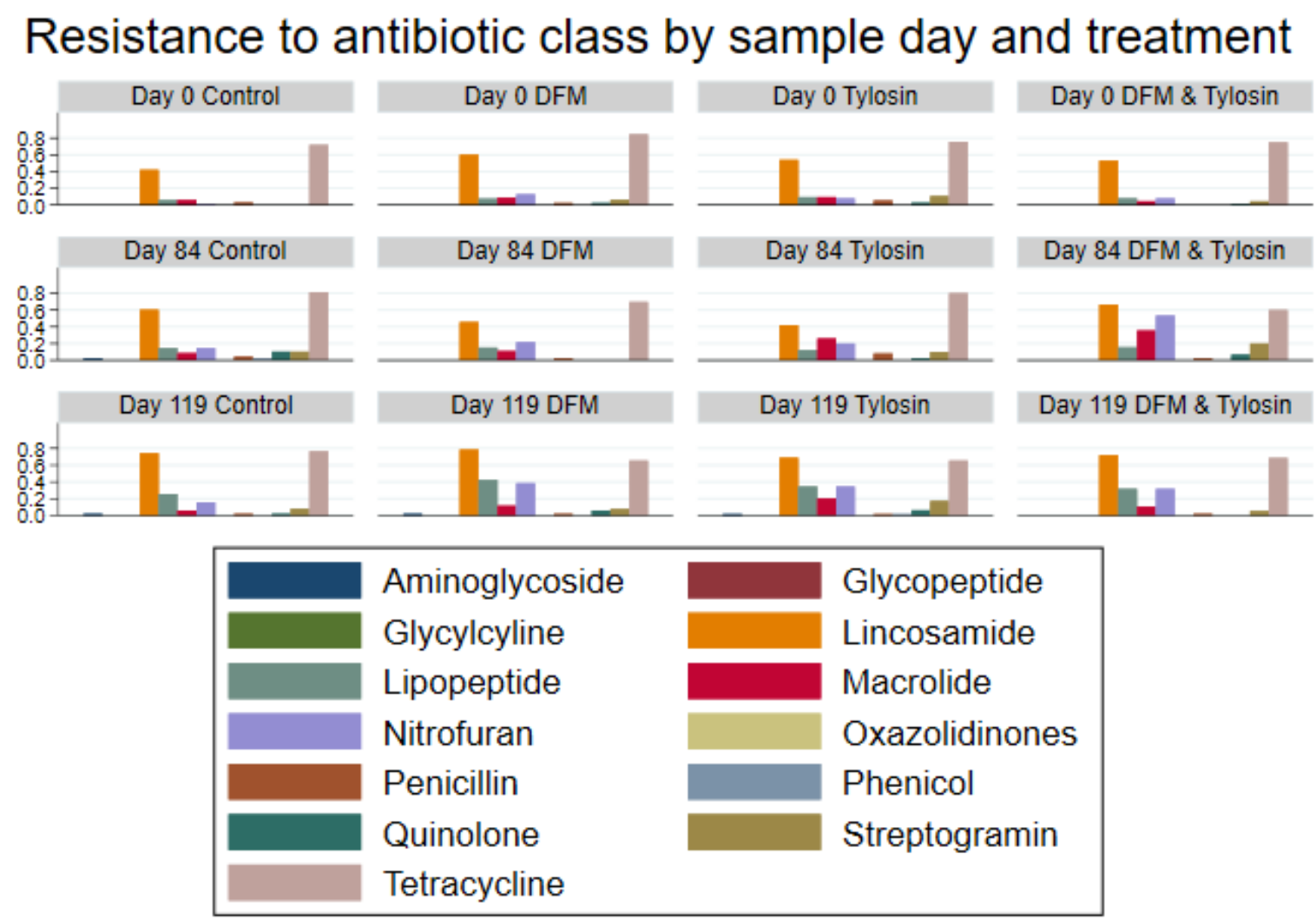

Figure 4 Resistance (proportion) of Enterococcus spp. isolates to each antibiotic class by sample day and treatment group. 


\begin{tabular}{|c|c|c|c|c|c|c|c|c|c|c|c|c|c|c|c|c|c|c|c|c|c|c|c|c|}
\hline & $\begin{array}{c}\# \\
\text { Resistant } \\
\text { (of } 693 \\
\text { tested) }\end{array}$ & $\begin{array}{c}\% \\
\text { Resistant }\end{array}$ & $\begin{array}{r}93 \\
\text { Conf } \\
\text { Inte }\end{array}$ & $\begin{array}{l}5 \% \\
\text { idence } \\
\text { erval }\end{array}$ & $\mid<0.015$ & 0.015 & 0.03 & 0.06 & 0.125 & 0.25 & 0.5 & 1 & 2 & 4 & 8 & 16 & 32 & 64 & 128 & 256 & 512 & 1024 & 2048 & $>2048$ \\
\hline Chloramphenicol & 2 & 0.3 & 0.03 & 1.0 & & & & & & & & & 1.73. & 65.22 & 32.32 & 0.435 & 0.145 & 0.145 & & & & & & \\
\hline Ciprofloxacin & 22 & 3.2 & 2 & 4.8 & & & & & 0.58 & 7.94 & 47.04 & 30.45 & 10.82 & 3.03 & 0.145 & & & & & & & & & \\
\hline Daptamycin & 124 & 17.9 & 15.1 & 21.0 & & & & & & 0.87 & 0.43 & 2.45 & 13.85 & 64.5 & 16.88 & 0.575 & 0.435 & & & & & & & \\
\hline Erythromycin & 80 & 11.5 & 9.26 & 14.2 & & & & & & 48.92 & 24.1 & 2.16 & 5.19 & 8.18 & 4.04 & 7.5 & & & & & & & & \\
\hline Gentamicin & 0 & 0.0 & 0 & $0.53^{*}$ & & & & & & & & & & & & & & & 100 & & & & & \\
\hline Kanamycin & 3 & 0.4 & \begin{tabular}{|l|}
0.09 \\
\end{tabular} & 1.3 & & & & & & & & & & & & & & & 83.26 & 14.43 & 1.88 & 0.435 & & \\
\hline Lincomycin & 413 & 59.6 & 55.8 & 63.3 & & & & & & & & 35.96 & 2.45 & 2.02 & 11.43 & 48.2 & & & & & & & & \\
\hline Linezolid & 0 & 0.0 & 0 & $0.53^{*}$ & & & & & & & 0.87 & 3.75 & 76.48 & 18.9 & & & & & & & & & & \\
\hline Nitrofurantoin & 141 & 20.4 & 17.4 & 23.5 & & & & & & & & & 1.3 & 0.29 & 0.435 & 18.9 & 28.72 & 30.01 & 20.35 & & & & & \\
\hline Penicillin & 20 & 2.9 & 1.77 & 4.4 & & & & & & 7.79 & 4.18 & 16.88 & 40.12 & 25.69 & 2.45 & 2.89 & & & & & & & & \\
\hline Streptomycin & 1 & 0.1 & 0 & 0.8 & & & & & & & & & & & & & & & & & 99.86 & 0.145 & & \\
\hline Quinupristin/Dalfopristin & 57 & 8.2 & 6.29 & 10.5 & & & & & & & 39.25 & 5.05 & 47.47 & 7.94 & 0.145 & 0.145 & & & & & & & & \\
\hline Tetracycline & 508 & 73.3 & 69.8 & 76.6 & & & & & & & & 22.37 & 1.88 & 0.87 & 1.59 & 3.17 & 18.04 & 52.09 & & & & & & \\
\hline Tigecycline & 0 & 0.0 & 0 & $0.53^{*}$ & & 0.58 & 17.75 & 26.7 & 53.1 & 1.88 & & & & & & & & & & & & & & \\
\hline Tylosin & 60 & 8.7 & \begin{tabular}{|l|}
6.67 \\
\end{tabular} & 11.0 & & & & & & 0.435 & 0.72 & 8.51 & 61.33 & 13.28 & 5.77 & 1.3 & 1.01 & 7.65 & & & & & & \\
\hline Vancomycin & 0 & 0.0 & 0 & $0.53^{*}$ & & & & & & 0.87 & 52.38 & 44.73 & 0.72 & 1.3 & & & & & & & & & & \\
\hline
\end{tabular}

${ }^{*} 97.5 \%$ One-sided CI

Figure 3 Percentage of Enterococcus spp. isolates that were resistant and their distribution across minimum inhibitory concentrations (MIC) for each antibiotic. Black vertical lines indicate the human CLSI (or, NARMS) interpretive breakpoint, grey boxes indicate areas above and below the highest and lowest limits of the assay antibiotic concentrations, respectively. Isolates which exceeded growth at the highest antibiotic concentration were placed in the next MIC column (shown in the grey area). 


\subsection{Multi-level mixed effects logistic regression modeling of antibiotic resistance phenotype}

A multi-level mixed effects logistic regression on tetracycline-resistant enterococci with binary endpoints showed a trend towards decreased resistance to tetracycline from Day 0 to Day 84 for the DFM and combination tylosin/DFM groups; however, this was not statistically significant $(\mathrm{P}>0.05)$. Additionally, there was decreased resistance to tetracycline in the tylosin group from Day 84 to Day 119 (following product withdrawal); however, these differences were also not significant (Figure 5).

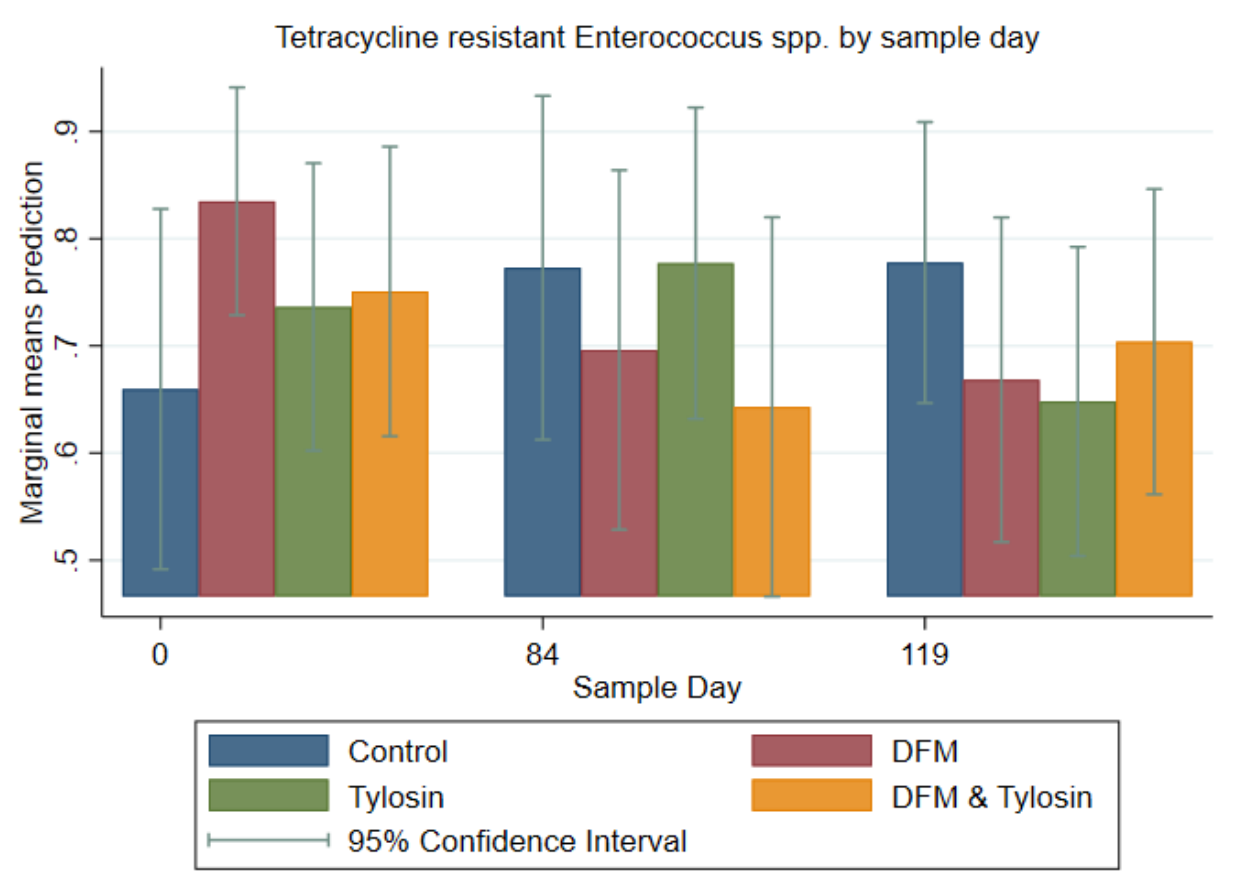

Figure 5 Marginal means with 95\% confidence intervals of a 2x2x2 multi-level mixed logistic regression model, using factors of DFM, tylosin, and sample day on the binary outcome of tetracycline resistant Enterococcus spp.

A multi-level mixed effects logistic regression on erythromycin-resistance among enterococci isolates with binary endpoints showed a trend of significantly $(\mathrm{P}<0.05)$ higher resistance to macrolides in the combination DFM/tylosin group on Day 84 compared to Day 0. Additionally, after the withdrawal of tylosin, resistance to macrolides significantly decreased in the combination group from Day 84 to Day 119 (Figure 6). The tylosin group showed a similar increase from Day 0 to Day 84; however, the differences were not statistically significant $(\mathrm{P}>0.05)$. 


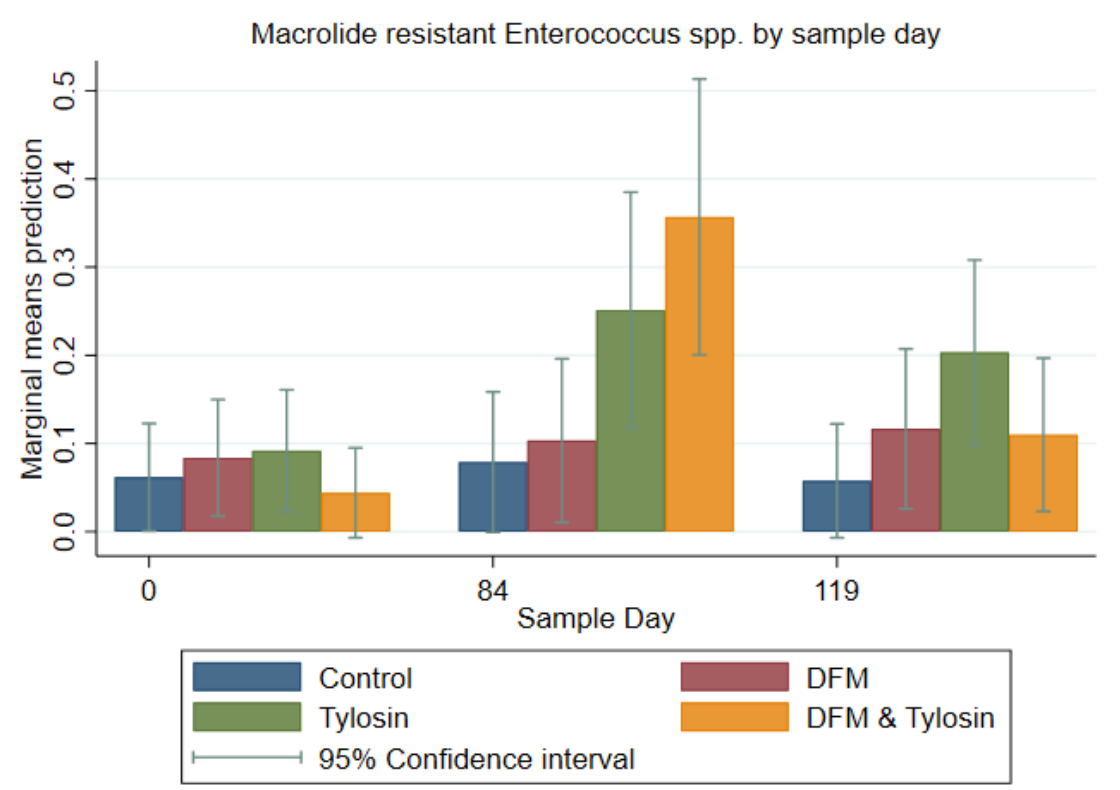

Figure 4 Marginal means with 95\% confidence intervals of a 2x2x2 multi-level mixed logistic regression model, using factors of DFM, tylosin, and sample day on the binary outcome of macrolide resistant Enterococcus spp.

\section{Discussion}

Overall, there was a significant difference $(\mathrm{P}<0.05)$ in quantification of enterococci between plain and erythromycin supplemented agar in the first replicate for those pens receiving tylosin alone. This difference was no longer significant $(\mathrm{P}>0.05), 4$ weeks after the withdrawal of tylosin at the end of the trial, and this effect was not present in any of the other groups, namely the combined tylosin/DFM group. This suggests the DFM may have had an attenuating effect on macrolide resistance when fed in conjunction with tylosin. Additionally, the isolates from tylosin/DFM group also had significantly more phenotypic resistance to macrolides on Day 84 when compared to Day 0, and less resistance was found at slaughter compared to Day 84 . This trend of increased resistance after a period of time being fed tylosin, then a decrease following its withdrawal, agrees with previous results from Beukers et al., [28] in that the withdrawal of tylosin prior to slaughter contributes to a decrease in macrolide-resistant enterococci.

A systematic review by Cazer et al. [19] concerning the effects of tylosin on antimicrobial resistance in beef cattle, found synthesized evidence that tylosin feeding increased the proportion of macrolide resistant enterococci in the gastrointestinal tract. When tylo$\sin$ is fed in conjunction with the ionophore monensin, enterococci have also been shown to exhibit increased resistance to macrolides [33]. However, it has been suggested that more than simple antibiotic use is at play, as a study by Jackson et al. showed that while macrolide-resistant Enterococcus spp. were higher on a farm which used tylosin, they were still present on another farm which did not use tylosin [34]. This implies that the environment must play an important role in sustaining resistance and its magnitude likely therefore likely reflects the scale of historical use. As suggested previously by Muller et al., in a study in which cattle that were not fed tylosin did not have significantly less macrolide resistant enterococci than cattle which were consistently fed tylosin. [35], both continuously and intermittently fed groups (and a negative control group) showed a significant increase in erythromycin resistance from arrival in the feedlot until late in the feeding period that could not be attributed to concurrent feeding of tylosin. 
Others have asserted that tylosin minimally affects resistance in beef cattle, and suggest that resistance may be seasonal; however, it should be noted that in the month in which the tylosin-treated cattle exhibited a higher prevalence of macrolide-resistant enterococci, the corresponding pen also had a higher prevalence of macrolide resistant Enterococcus spp. [36]. It was of interest in our study that the DFM was associated with decreased tetracycline resistance from Day 0 to Day 84, which occurred in both quantification and phenotypic resistance among isolates, though it was not significant.

A study by Amachawadi et al. [37] pointed out the potential problems with using commercial probiotics, including the isolation of multidrug-resistant E. faecium from the products. Even though the probiotic used in this study was not multidrug-resistant, it could still have significant impacts on antimicrobial resistance, be subjected to selection pressures, or conjugate plasmids with resistant bacteria, as Enterococcus faecium is known to readily share plasmids with Staphylococcus aureus [38] as well as members of its own genus or species. Therefore, the significantly decreased difference (and therefore increased resistance) shown in replicate 1 in macrolide resistance in the DFM group as compared to the tylosin group alone could imply the DFM may mitigate this resistance among both $\log _{10}$ CFU per gram of feces quantification and enterococcal isolates. Additionally, the lack of a significantly decreased difference in macrolide resistance from Day 0 to Day 112 in both the DFM group and the combined DFM/tylosin group may suggest the combination of a macrolide-susceptible probiotic, withdrawal of tylosin before slaughter, and movement of cattle may be a viable future alternative to combat erythromycin resistance in beef cattle fed tylosin.

\section{Conclusions}

In conclusion, tylosin and its subsequent withdrawal have a measurable effect on macrolide-resistant enterococci during the cattle feeding period, and macrolide resistance among isolates, which agrees with previous studies. While the results in our first replicate favored the use of a probiotic to mitigate erythromycin resistance among fecal enterococci, the results from the second replicate were inconclusive. Starting levels (Day 0) of macrolide resistance can differ between trial replicates, and whenever cattle spend extended periods of time in any feedlot environment the levels of resistance will rise uniformly across all groups, thus reducing the potential for differences to emerge between treatment groups. Thus, trial Replicate 1 may have differed from trial Replicate 2. Meanwhile, other factors, such as season or age of cattle age may also be at play. Therefore, further studies to evaluate the use of an Enterococcus-based probiotic while accounting for pre-existing environmental conditions should be performed.

Author Contributions: Data curation, Sarah Murray and Harvey Scott; Formal analysis, Sarah Mur-
ray, Keri Norman and Harvey Scott; Funding acquisition, Jason Sawyer and Harvey Scott; Investi-
gation, Sarah Murray, Ashlyn Holbert, Keri Norman, Sara Lawhon, Jason Sawyer and Harvey Scott;
Methodology, Ashlyn Holbert, Keri Norman, Sara Lawhon and Jason Sawyer; Project administra-
tion, Harvey Scott; Supervision, Jason Sawyer and Harvey Scott; Writing - original draft, Sarah
Murray; Writing - review \& editing, Keri Norman, Sara Lawhon and Harvey Scott. All authors have
read and agreed to the published version of the manuscript.

Funding: The data presented in this publication were derived from a project funded by USDANIFA-AFRI 2016-68003-24607 entitled "Voluntary compliance in antimicrobial stewardship programs: a critical factor for effective intervention". Any recommendations, opinions, findings or conclusions expressed in this publication are those of the publishing authors and do not necessarily represent those of the United States Department of Agriculture.

Institutional Animal Care and Use Committee Statement: The study was approved by the Institutional Animal Care and Use Committee of Texas A\&M University (Animal Use Protocol \#2015026A)

Data Availability Statement: The datasets used and/or analyzed are available from the corresponding author on reasonable request. 

Acknowledgments: Thanks to the Microbial Ecology and Molecular Epidemiology $\left(\mathrm{ME}^{2}\right)$ laboratory
members at Texas A \& M University for assistance with sample processing. Additionally, we would
like to thank the staff at the McGregor Texas Agrilife Research Center for their service during this
trial.

Conflicts of Interest: The authors declare no conflicts of interest in this study.
101 vaccine approaches to control in feedlot cattle. J Anim Sci 2016, 94, 1620-1632, doi:10.2527/jas.2015-0261.

8. Nagaraja, T.G.; Chengappa, M.M. Liver abscesses in feedlot cattle: a review. J Anim Sci 1998, 76, 287-298, doi:10.2527/1998.761287x.

9. Nagaraja, T.G.; Beharka, A.B.; Chengappa, M.M.; Carroll, L.H.; Raun, A.P.; Laudert, S.B.; Parrott, J.C. Bacterial flora of liver abscesses in feedlot cattle fed tylosin or no tylosin. J Anim Sci 1999, 77, 973-978, doi:10.2527/1999.774973x.

10. Amachawadi, R.G.; Scott, H.M.; Aperce, C.; Vinasco, J.; Drouillard, J.S.; Nagaraja, T.G. Effects of in-feed copper and tylosin supplementations on copper and antimicrobial resistance in faecal enterococci of feedlot cattle. J Appl Microbiol 2015, 118, 1287-1297, doi:10.1111/jam.12790.

11. Brown, T.R.; Lawrence, T.E. Association of liver abnormalities with carcass grading performance and value. J Anim Sci 2010, 88, 4037-4043, doi:10.2527/jas.2010-3219.

12. Nagaraja, T.G.; Lechtenberg, K.F. Liver Abscesses in Feedlot Cattle. Veterinary Clinics of North America: Food Animal Practice 2007, 23, 351-369, doi:https://doi.org/10.1016/j.cvfa.2007.05.002.

13. Brink, D.R.; Lowry, S.R.; Stock, R.A.; Parrott, J.C. Severity of liver abscesses and efficiency of feed utilization of feedlot cattle. J Anim Sci 1990, 68, 1201-1207, doi:10.2527/1990.6851201x.

14. Brown, H.; Bing, R.F.; Grueter, H.P.; McAskill, J.W.; Cooley, C.O.; Rathmacher, R.P. Tylosin and chloretetracycline for the prevention of liver abscesses, improved weight gains and feed efficiency in feedlot cattle. J Anim Sci 1975, 40, 207-213, doi:10.2527/jas1975.402207x.

15. Wileman, B.W.; Thomson, D.U.; Reinhardt, C.D.; Renter, D.G. Analysis of modern technologies commonly used in beef cattle production: conventional beef production versus nonconventional production using meta-analysis. J Anim Sci 2009, 87, 3418-3426, doi:10.2527/jas.2009-1778.

16. Aarestrup, F.M.; Carstensen, B. Effect of tylosin used as a growth promoter on the occurrence of macrolide-resistant enterococci and staphylococci in pigs. Microb Drug Resist 1998, 4, 307-312, doi:10.1089/mdr.1998.4.307.

17. Jacob, M.E.; Fox, J.T.; Narayanan, S.K.; Drouillard, J.S.; Renter, D.G.; Nagaraja, T.G. Effects of feeding wet corn distillers grains with solubles with or without monensin and tylosin on the prevalence and antimicrobial susceptibilities of fecal foodborne pathogenic and commensal bacteria in feedlot cattle. J Anim Sci 2008, 86, 1182-1190, doi:10.2527/jas.2007-0091. 
18. Zaheer, R.; Cook, S.R.; Klima, C.L.; Stanford, K.; Alexander, T.; Topp, E.; Read, R.R.; McAllister, T.A. Effect of subtherapeutic vs. therapeutic administration of macrolides on antimicrobial resistance in Mannheimia haemolytica and enterococci isolated from beef cattle. Front Microbiol 2013, 4, 133, doi:10.3389/fmicb.2013.00133.

19. Cazer, C.L.; Eldermire, E.R.B.; Lhermie, G.; Murray, S.A.; Scott, H.M.; Gröhn, Y.T. The effect of tylosin on antimicrobial resistance in beef cattle enteric bacteria: A systematic review and meta-analysis. Prev Vet Med 2020, 176, 104934, doi:10.1016/j.prevetmed.2020.104934.

20. Zaheer, R.; Cook, S.R.; Barbieri, R.; Goji, N.; Cameron, A.; Petkau, A.; Polo, R.O.; Tymensen, L.; Stamm, C.; Song, J.; et al. Surveillance of Enterococcus spp. reveals distinct species and antimicrobial resistance diversity across a One-Health continuum. Scientific Reports 2020, 10, 3937, doi:10.1038/s41598-020-61002-5.

21. Emori, T.G.; Gaynes, R.P. An overview of nosocomial infections, including the role of the microbiology laboratory. Clin Microbiol Rev 1993, 6, 428-442, doi:10.1128/cmr.6.4.428.

22. Arias, C.A.; Murray, B.E. Emergence and management of drug-resistant enterococcal infections. Expert Review of Antiinfective Therapy 2008, 6, 637-655, doi:10.1586/14787210.6.5.637.

23. Arias, C.A.; Murray, B.E. The rise of the Enterococcus: beyond vancomycin resistance. Nat Rev Microbiol 2012, 10, 266-278, doi:10.1038/nrmicro2761.

24. Huycke, M.M.; Sahm, D.F.; Gilmore, M.S. Multiple-drug resistant enterococci: the nature of the problem and an agenda for the future. Emerg Infect Dis 1998, 4, 239-249, doi:10.3201/eid0402.980211.

25. Lebreton, F.; van Schaik, W.; Manson McGuire, A.; Godfrey, P.; Griggs, A.; Mazumdar, V.; Corander, J.; Cheng, L.; Saif, S.; Young, S.; et al. Emergence of Epidemic Multidrug-Resistant Enterococcus faecium from Animal and Commensal Strains. mBio 2013, 4, e00534-00513, doi:10.1128/mBio.00534-13.

26. Chen, J.; Fluharty, F.L.; St-Pierre, N.; Morrison, M.; Yu, Z. Technical note: Occurrence in fecal microbiota of genes conferring resistance to both macrolide-lincosamide-streptogramin B and tetracyclines concomitant with feeding of beef cattle with tylosin. J Anim Sci 2008, 86, 2385-2391, doi:10.2527/jas.2007-0705.

27. WHO. Critically important antimicrobials for human medicine. 2019.

28. Beukers, A.G.; Zaheer, R.; Cook, S.R.; Stanford, K.; Chaves, A.V.; Ward, M.P.; McAllister, T.A. Effect of in-feed administration and withdrawal of tylosin phosphate on antibiotic resistance in enterococci isolated from feedlot steers. Front Microbiol 2015, 6, 483, doi:10.3389/fmicb.2015.00483.

29. Müller, H.C.; Van Bibber-Krueger, C.L.; Ogunrinu, O.J.; Amachawadi, R.G.; Scott, H.M.; Drouillard, J.S. Effects of intermittent feeding of tylosin phosphate during the finishing period on feedlot performance, carcass characteristics, antimicrobial resistance, and incidence and severity of liver abscesses in steers. J Anim Sci 2018, 96, 2877-2885, doi:10.1093/jas/sky166.

30. Huebner, K.L.; Martin, J.N.; Weissend, C.J.; Holzer, K.L.; Parker, J.K.; Lakin, S.M.; Doster, E.; Weinroth, M.D.; Abdo, Z.; Woerner, D.R.; et al. Effects of a Saccharomyces cerevisiae fermentation product on liver abscesses, fecal microbiome, and resistome in feedlot cattle raised without antibiotics. Scientific Reports 2019, 9, 2559, doi:10.1038/s41598-019-39181-7.

31. Izquierdo, E.; Marchioni, E.; Aoude-Werner, D.; Hasselmann, C.; Ennahar, S. Smearing of soft cheese with Enterococcus faecium WHE 81, a multi-bacteriocin producer, against Listeria monocytogenes. Food Microbiol 2009, 26, 16-20, doi:10.1016/j.fm.2008.08.002.

32. CLSI. Performance Standards for Antimicrobial Susceptibility Testing. 2020, 30th ed CLSI supplement M100.

33. Jacob, M.E.; Fox, J.T.; Narayanan, S.K.; Drouillard, J.S.; Renter, D.G.; Nagaraja, T.G. Effects of feeding wet corn distillers grains with solubles with or without monensin and tylosin on the prevalence and antimicrobial susceptibilities of fecal foodborne pathogenic and commensal bacteria in feedlot cattle1. J Anim Sci 2008, 86, 1182-1190, doi:10.2527/jas.2007-0091.

34. Jackson, C.R.; Fedorka-Cray, P.J.; Barrett, J.B.; Ladely, S.R. Effects of tylosin use on erythromycin resistance in enterococci isolated from swine. Applied and environmental microbiology 2004, 70, 4205-4210, doi:10.1128/AEM.70.7.4205-4210.2004. 
35. Müller, H.C.; Van Bibber-Krueger, C.L.; Ogunrinu, O.J.; Amachawadi, R.G.; Scott, H.M.; Drouillard, J.S. Effects of intermittent feeding of tylosin phosphate during the finishing period on feedlot performance, carcass characteristics, antimicrobial resistance, and incidence and severity of liver abscesses in steers1. J Anim Sci 2018, 96, 2877-2885, doi:10.1093/jas/sky166.

36. Schmidt, J.W.; Vikram, A.; Miller, E.; Jones, S.A.; Arthur, T.M. In-Feed Tylosin Phosphate Administration to Feedlot Cattle Minimally Affects Antimicrobial Resistance. J Food Prot 2020, 350-364, doi:10.4315/0362-028x.Jfp-19-342.

37. Amachawadi, R.; Giok, F.; Shi, X.; Soto, J.; Narayanan, S.; Tokach, M.; Apley, M.; Nagaraja, T. Antimicrobial resistance of Enterococcus faecium strains isolated from commercial probiotic products used in cattle and swine. J Anim Sci 2018, 96, 912920, doi:10.1093/jas/sky056.

38. Clewell, D.B.; An, F.Y.; White, B.A.; Gawron-Burke, C. Sex pheromones and plasmid transfer in Streptococcus faecalis: a 